Rev Soc Esp Dolor

2014; 21(5): 295-296

\section{Utilidad de la radiofrecuencia pulsada en el dolor postoracotomía}

\section{Sr. Director:}

El dolor postoracotomía es un síntoma de alta incidencia que actúa como factor de riesgo de complicaciones postoperatorias (1) como hipoventilación, atelectasias y neumonía. A pesar de ello no existen tratamientos específicos. Describimos el caso de una paciente con dolor neuropático postoracotomía.

\section{CASO CLÍNICO}

Paciente mujer de 40 años, antecedentes personales: ansiedad, uveítis, HLA B-27 +, migrañas, bruxismo, gastritis, fumadora de 10 cigarrillos/día. Tratamiento lormetazepam $1 \mathrm{c} / 24 \mathrm{~h}$. Intervenida de incidentaloma renal izquierdo mediante lumbotomía con sección de $11^{\mathrm{a}}$ costilla y dolor neuropático postoracotomía en territorio nervio intercostal T11, con mal control analgésico.

Decidimos infiltrar espacios intercostales T10-T11 y T11-T12 con bupivacaína $0,5 \%+40 \mathrm{mg}$ de Trigon ${ }^{\circledR}, 5 \mathrm{ml}$ en cada espacio, EVA 1/10. Iniciamos tratamiento con Lyrica $^{\circledR} 75 \mathrm{mg}$, más Rivotril@ $0,5 \mathrm{mg} / 24 \mathrm{~h}$ y parches de Versatis ${ }^{\circledR} 5$ \%. Si dolor, tramadol 10-15 gotas cada 8 h. En la siguiente revisión a los 10 días, EVA 5/10. Repetimos infiltración y mantenemos Lyrica $^{\circledR}$ en pauta ascendente, con EVA post infiltración 1/10. Suspendemos parches de Versatis ${ }^{\circledR} 5 \%$, por falta de efecto.

La programamos para radiofrecuencia pulsada (RFP) en espacios intercostales T10-T11 y T11-T12, previa infiltración con anestésico local, bupivacaína $0,5 \%$ más dexametasona $4 \mathrm{mg}, 5 \mathrm{ml}$ en cada espacio. Parámetros de estimulación son 45 Hz y 120 segundos (Fig. 1). Repetimos procedimiento al mes.

Diez meses después, se encuentra asintomática (EVA0/10).

\section{DISCUSIÓN}

La cronificación del dolor neuropático postoracotomía alcanza una prevalencia de hasta el $50 \%$ de los pacientes, llegando a ser incapacitante en el $5 \%$ de los casos. Persiste en el $30 \%$ a los 5 años, por eso es tan importante su manejo postoperatorio. En su tratamiento se requiere analgesia multimodal, aunque su mejor tratamiento es preventivo (2).
Las técnicas intervencionistas (3) están indicadas en pacientes con dolor torácico irradiado con patrón segmentario, que no responden al tratamiento conservador y con respuesta temporal al bloqueo del nervio intercostal de las ramas anteriores, a nivel de ángulo costal, entre 7 y $10 \mathrm{~cm}$ de las apófisis espinosas. Para ello retraemos hacia arriba la piel que cubre el borde inferior de la costilla e introducimos la aguja con un ángulo de $20^{\circ}$ hasta alcanzar el hueso. Posteriormente, se endereza la aguja y se desliza bajo el reborde costal inferior, avanzando 2-3 mm (4). Se aconseja bloquear dos niveles por encima de la lesión y tres por debajo de la misma (5), aunque en nuestro caso con el bloqueo de dos niveles fue suficiente.

Otros autores recomiendan radiofrecuencia adyacente al ganglio de la raíz dorsal. En los segmentos situados por debajo de T7, como es nuestro caso, se utiliza la siguiente técnica. Se inserta una cánula de $100 \mathrm{~mm}, 1 \mathrm{~cm}$ medial al ángulo de las costillas. Se avanza cuidadosamente siguiendo el ángulo paralelo a las costillas hasta que se alcanza la parte craneodorsal del foramen intervertebral. El estilete de la cánula se reemplaza por el electrodo de radiofrecuencia y se hace una estimulación a $50 \mathrm{~Hz}$. Se considera una correcta posición cuando el paciente percibe una sensación de hormigueo en el dermatoma seleccionado con un estímulo de 0,4-1 V. Posteriormente se realiza una estimulación a $2 \mathrm{~Hz}$, para descartar posición intraganglionar (6).

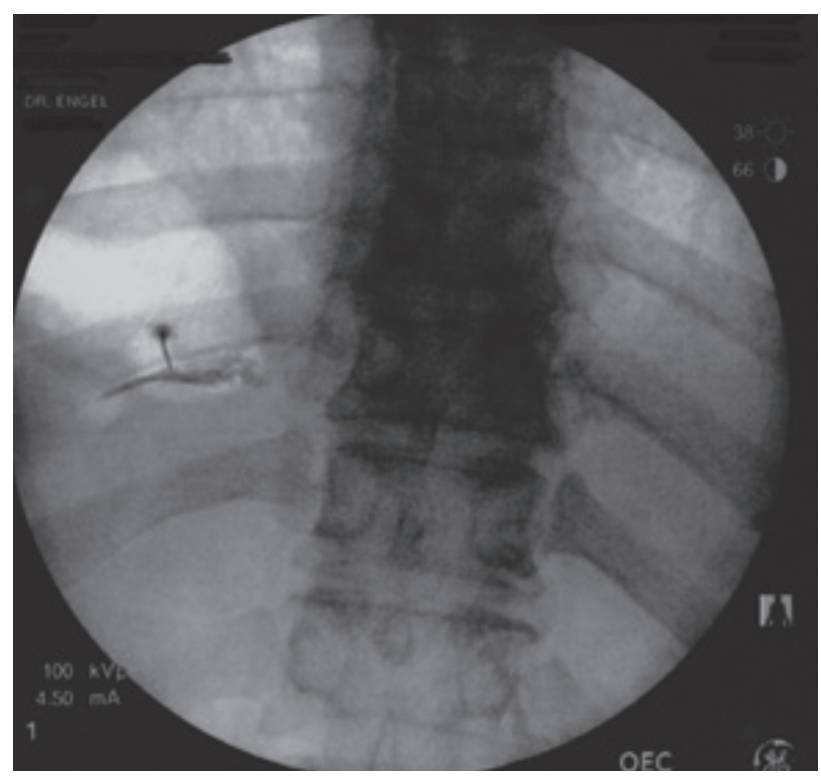

Fig. 1. Radiofrecuencia pulsada nervio intercostal. Contraste. 
Las tasas de éxito de la radiofrecuencia varían entre el 50 y el $85 \%$. Y, en cuanto a los efectos secundarios, puede aparecer dolor quemante e hipoestesias en el dermatoma tratado en el $17 \%$ de los pacientes, que suelen desaparecer al cabo de unas semanas (7).

\section{García Huerta y J.M. Lozano Enguita}

Servicio de Anestesiología. Hospital Santa Bárbara. Soria

\section{BIBLIOGRAFÍA}

1. García-Tirado J, Rieger-Reyes C. Técnicas de cierre de la toracotomía y su relación con el dolor postoracotomía: revisión sistemática. Arch Bronconeumol 2012;48:22-8.
2. Treede RD, Jensen TS, Campbell JN, Cruccu G, Dostrovsky JO, Griffin JW, et al. Neuropathic pain: Redefinition and a grading system for clinical and research purposes. Neurology 2008;70:1630-5.

3. Cohen SP, Sireci A, Wu CL, Larking TM, Williams KA, Hurley RW. Pulsed radiofrequency of the dorsal root ganglia is superior to pharmacotherapy or pulsed radiofrequency of the intercostal nerves in the treatment of chronic postsurgical thoracic pain. Pain Physician 2006;9:227-35.

4. Abejón-González D, Pérez-Cajaraville J. Manual práctico dolor neuropático. Barcelona: Elsevier España, S.L.; 2010. p. 189-209.

5. Savage C, McQuitty C, Wang D, Zwischenberger JB. Postthoracotomy pain management. Chest Surg Clin NAm 2002;12:251-63.

6. López-Rodríguez MA, Varela M, Camba MA. Aplicaciones de la radiofrecuencia en el tratamiento del dolor crónico benigno. Una revisión de las publicaciones de los últimos 6 años. Rev Soc Esp Dolor 2001;8:397-411.

7. Detterbeck FC. Efficacy of methods of intercostal nerve blockade for pain relief after thoracotomy. Ann Thorac Surg 2005;80:1550-9. 